\title{
STATISTICAL CONVERGENCE IN 2-METRIC SPACES
}

\author{
FATIH NURAY
}

Abstract. In this paper, notions of statistical convergence, statistically Cauchy sequence, $\mathscr{N}_{\theta}$ and lacunary statistical convergence in 2-metric space will be introduced. Also some inclusion relations between these concepts will investigated.

Mathematics subject classification (2010): 40A05, 47H10.

Keywords and phrases: Statistical convergence, lacunary sequence, 2-metric spaces.

\section{REFERENCES}

[1] A. Aliouche, C. Simpson, Fixed points and lines in 2-metric spaces, Adv. Math. 229 (2012) no. 1, 668-690.

[2] F. BAşAR, Summability Theory and its Applications, Bentham Science Publishers, İstanbul, eISBN: 978-1-60805-252-3, 2012.

[3] J. CONnOR, The statistical and strong p-Cesàro convergence of sequences, Analysis 8 (1988), 47-63.

[4] J. Connor, K. G. Grosse-Erdmann, Sequential definitions of continuity for real functions, Rocky Mountain J. Math. 33 (2003) no. 1, 93-121.

[5] H. FAST, Sur la convergence statistique, Colloq. Math., 2 (1951) 241-244.

[6] A. R. Freedman, J. J. Sember, M. Raphael, Some Cesàro-Type Summability Spaces, Proc. Lond. Math. Soc., 37 (1978), no. 3, 508-520.

[7] R. FreEse, Y. CHo, Geometry of linear 2-normed spaces, Nova Science Publishers, Hauppauge, NY (2001).

[8] J. A. Fridy, C. ORHAN, Lacunary statistical summability, J. Math. Anal. Appl., 173(1993), $497-504$.

[9] J. A. Fridy, C. ORhAN, Lacunary statistical convergence, Pacific J. Math., 160 (1993), 43-51.

[10] J. A. FRIDY, On statistical convergence, Analysis, 5 (1985) 301-313.

[11] S. GÄHLER, 2-metric Raume and ihre topologische strucktur, Math. Nachr., 26 (1963), 115-148.

[12] S. GÄHLER, Uber die unifromisieberkeit 2-metrischer Raume, Math.Nachr., 28 (1965), $235-244$.

[13] G. H. Hardy, E. M. Wright, An Introduction to the Theory of Numbers, fourth ed., Oxford, 1960.

[14] K. IsÉKI, Fixed point theorems in 2-metric spaces, Math. Seminar Notes XIX (1975).

[15] M. KHAn, On fixed point theorems in 2-metric space, Publ. de l'Institute Mathématique, 41 (1980), $107-112$.

[16] B. K. LAhiRI, P. DAS, L. K. DEY, Cantor's theorem in 2-metric spaces and its applications to fixed point theorems, Taiwanese Journal of Mathematics 15 (2011), no. 1, 337-352.

[17] Z. LiU, F. ZHANG, Characterization of common fixed points in 2-metric spaces, Rostock Math. Kolloq., 55 (2001), 49-64.

[18] M. Mursaleen, F. BASAR, Sequence Spaces: Topics in Modern Summability Theory, CRC Press, Taylor and Francis Group, Series: Mathematics and Its Applications, Boca Raton, London, New York, 2020.

[19] S. V. R. NAIDU, J. R. Prasad, Fixed point theorem in 2-metric spaces, Indian J Pure Appl Math., 17 (1986), 974-93.

[20] S. V. R. NAIDU, Some fixed point theorems in metric and 2-metric spaces, Int. J. Math. Math. Sci., 28 (2001), 625-636.

[21] B. RHOADES, Contraction type mappings on a 2-metric space, Math. Nachr., 91 (1979), 151-155.

[22] I. J. SCHOENBERG, The integrability of certain functions and related summability methods, Amer. Math. Monthly, 66 (1959), 361-375. 
[23] S. L. Singh, B. TiWARI, V. GuPTA, Common fixed points of commuting mappings in 2-metric spaces and an application, Math. Nachr., 95 (1980), 293-297.

[24] A. White, 2-Banach spaces, Math. Nachr., 42 (1976), 43-60. 